\title{
Retromer and its role in regulating signaling at endosomes
}

\author{
Matthew N.J. Seaman \\ University of Cambridge \\ Cambridge Institute for Medical Research \\ Wellcome Trust/MRC Building \\ Addenbrookes Hospital \\ Cambridge \\ CB2 OXY
}

\begin{abstract}
The retromer complex is a key element of the endosomal protein sorting machinery being involved in trafficking of proteins from endosomes to the Golgi and also endosomes to the cell surface. There is now accumulating evidence that retromer also has a prominent role in regulating the activity of many diverse signaling proteins that traffic through endosomes and this activity has profound implications for the functioning of many different cell and tissue types from neuronal cells to cells of the immune system to specialized polarized epithelial cells of the retina. In this review the protein composition of the retromer will be described along with many of the accessory factors that facilitate retromer-mediated endosomal protein sorting to detail how retromer activity contributes to the regulation of several distinct signaling pathways.
\end{abstract}


Introduction - retromer and endosomal protein sorting

Signaling events initiated at the plasma membrane by a receptor binding its respective ligand may be modulated by the receptor being endocytosed and then delivered to an endosome. A classical example of this process is the down regulation of the epidermal growth factor receptor (EGFR) after binding of ligand, ubiquitylation and uptake into clathrin-coated vesicles. Following endocytosis and arrival at a structure termed a signaling endosome the activation of the tyrosine kinase domain of the EGFR can occur. Once in an endosome, the activated EGFR is sorted by the ESCRT (Endosome sorting complex required for transport) machinery into nascent intralumenal vesicles which results in the EGFR being effectively removed from the cytoplasm of the cell silencing the active tyrosine kinase in the cytoplasmic tail of the EGFR. The subsequent proteolytic destruction of the EGFR in a lysosome finishes the task of modulating the signaling cascade initiated by ligand binding at the cell surface (for review see Schuh and Audhya 2014). It is noteworthy that some cancers have been linked to mutations in the ESCRT-1 complex that operates to recognize the ubiquitin tag added to the activated EGFR underscoring the biological importance of endosomally-localized sorting machinery in signal modulation. It is also now well established that other receptor tyrosine kinases (RTKs) such as the platelet-derived growth factor receptor (PDGFR) and insulin receptor (IR) are similarly silenced by ESCRT-mediated sorting into endosomal intralumenal vesicles.

Whilst the role of ESCRT proteins in modulating signaling is now well established and has been covered in depth in other reviews, there is now growing evidence of the importance of additional endosomally-localised sorting machinery - namely the retromer complex - in the modulation of signaling events. The retromer complex was first identified several years ago from studies in yeast focused on the endosome-to-Golgi pathway. The yeast retromer complex was shown to mediate the retrieval of a vacuolar hydrolase receptor protein, Vps10p from endosomes to the Golgi complex. The yeast retromer complex was revealed to comprise five proteins, all encoded by vacuolar protein sorting (VPS) genes, that assemble to form a stable 
heteropentamer (Seaman et al., 1997; Seaman et al., 1998). The five retromer proteins are: Vps35p, Vps29p, Vps26p, Vps17p and Vps5p. It has been shown that Vps35p forms a stable trimer with Vps29p and Vps26p which functions in selecting membrane proteins, e.g. Vps10p (cargo) for retrieval to the Golgi. The Vps5p and Vps17p are both members of the sorting nexin (SNX) family and form a stable dimer that can assemble on membranes to drive tubulation (Horazdovsky et al., 1997). The ability of the Vps5p-Vps17p dimer to tubulate membranes is derived from their carboxy-terminal BinAmphiphysin-Rvs (BAR) domains and hence Vps5p and Vps17p are referred to as SNX-BAR proteins (reviewed in Van Weering and Cullen, 2014). Loss of any of the retromer components results in a defect in endosome-to-Golgi retrieval and can also lead to instability and rapid degradation of the remaining proteins.

The retromer complex is conserved in all eukaryotes and in mammalian cells a cargo-selective trimer of VPS35, VPS29 and VPS26 operates with a sorting nexin dimer (containing SNX-BAR proteins) of SNX1 or SNX2 paired with either SNX5 or SNX6 to mediate the retrieval of various proteins from endosomes to the Golgi including lysosomal hydrolase receptors such as the cation-independent mannose 6-phosphate receptor (CIMPR) and the Vps10p homologue, sortilin (Renfrew-Haft et al., 2000; Seaman, 2004; Arighi et al., 2004; Carlton et al., 2004). It is worth noting however that in higher eukaryotes such as humans, the cargo-selective trimer forms only a loose association with the sorting nexin dimer (Swarbrick et al., 2011; Harbour and Seaman, 2011) and thus the mechanisms that govern endosome-to-Golgi retrieval may be subtly different from yeast to humans. One major difference between yeast and humans is that, in humans and other higher eukaryotes, retromer has a prominent role to play in the endosome-to-cell surface retrieval pathway. This is because the mammalian retromer complex is able to associate with a host of additional 'accessory' factors that play a key role in endosomal protein sorting, especially the endosome-to-cell surface pathway (reviewed in Seaman, 2012). This means that the retromer complex can regulate the cell surface levels of many different proteins, some of which function as signaling receptors. Recent data has also implicated retromer in 
directly regulating the activity of signaling receptors by displacing key signaling intermediates from the cytoplasmic domains of signaling receptors.

VPS26 - an $\alpha$-arrestin

The first clues that retromer may play a direct role in regulating signaling events came from the determination of the structure of the VPS26 protein. Once the crystal structure of VPS26 was solved through x-ray diffraction, it was realized that VPS26 was structurally highly related to the $\beta$-arrestin family of proteins (Shi et al., 2006; Collins et al., 2008). The similarity of VPS26 to the $\beta$-arrestin family of proteins was such that VPS26 has been termed an $\alpha$ arrestin (for reviews see Aubry and Klein, 2013; Gurevich and Gurevich, 2014). The $\beta$-arrestin proteins are primarily involved in the uptake of activated G-protein coupled receptors (GPCRs) by linking GPCRs to the endocytic machinery that forms clathrin-coated pits at the cell surface. Once GPCRs have reached an endosome, their signaling activity is down regulated and they are then recycled to the cell surface. The recycling of the $\beta 2$-adrenergic receptor - a classical GPCR - from endosomes to the cell surface requires the function of a sorting nexin protein, specifically SNX27 in conjunction with retromer (Temkin et al., 2011). Importantly, SNX27 differs from the SNX-BAR proteins (e.g. SNX1 and SNX2) by lacking a BAR domain and thus is not believed to mediate membrane tubulation. Although lacking a BAR domain, SNX27 does posses other functional domains, namely a PDZ (PSD95, Dlg1, zo-1)-binding domain and a FERM (4.1, ezrin, radixin, moesin) domain - both of which have important roles in how SNX27 links retromer to signaling proteins.

Retromer accessory proteins: the WASH complex and SNX27

The SNX27 protein associates with the retromer cargo-selective trimer by binding to VPS26 (Gallon et al., 2014). In addition to binding to VPS26, the SNX27 protein also interacts with a protein called FAM21 (also now known as WASHC2) which is a component of the WASH complex (Temkin et al., 2011; 
Freeman et al., 2014). Like retromer, the WASH complex is comprised of five proteins, namely WASH1, KIAA1033 (now WASHC3), strumpellin (now WASHC4), CCDC53 (now WASHC5) and FAM21 (Derivery et al., 2009) but unlike retromer, the WASH complex is not universally conserved being absent in many organisms including yeast. The WASH complex mediates formation of branched filamentous (F-) actin on endosomes and is required for sorting many endosomal proteins to their destination but is generally more prominently required for endosome-to-cell surface recycling (see Seaman et al., (2013) for review). Indeed it has been reported that FAM21 can prevent the endosome-to-Golgi retrieval of proteins such as the Glut-1 glucose transporter and direct it into a SNX27-retromer-mediated endosome-to-cell surface pathway (Lee et al., 2016). In mammals, the WASH complex is recruited to endosomes by the direct interaction of FAM21 with VPS35 (Harbour et al., 2012; Jia et al., 2012; Helfer et al., 2013).

Thus there is a complex and dynamic network of protein-protein interactions that underlie the mechanism of endosome-to-cell surface recycling and revolve around the cargo-selective retromer trimer of VPS35-VPS29-VPS26 (see Figure 1). One way retromer may, through the WASH complex, contribute to regulating signaling events on endosomes is through the creation of actin-stabilised microdomains that potentiate signaling initiated by specific receptors. This suggestion, whilst conceptually attractive, currently lacks strong experimental evidence.

The importance of the retromer trimer in regulating GPCR signaling goes beyond that of providing binding sites for the WASH complex and SNX27. The VPS26 protein can, due to its similarity to $\beta$-arrestin, displace $\beta$-arrestin from certain GPCRs and thereby modulate the signaling activity. For the parathyroid hormone receptor (PTHR), activation by ligand binding whilst at the cell surface triggers the production of cyclic AMP (CAMP) and results in binding of the PTHR by $\beta$-arrestin. Following endocytosis of the PTHR with $\beta$ arrestin, the production of cyclic adenosine monophosphate (cAMP) continues until the $\beta$-arrestin is displaced from the cytoplasmic domain of the PTHR by 
retromer - an action believed to be driven by VPS26 (Feinstein et al., 2011). Subsequently the PTHR is recycled back to the cell surface through an association with SNX27 mediated through the PDZ domain of SNX27 binding to a PDZ ligand in the cytoplasmic domain of the PTHR (Chan et al., 2016; McGarvey et al., 2016). This process effectively silences the signaling activity of the PTHR and 'resets' the system ready for another round of ligandbinding, receptor activation and signaling and thus is quite different to the down regulation of, for example, the EGFR by the ESCRT complex.

The role of retromer and its associated proteins in regulating the activity and localization of GPCRs such as the $\beta 2$-adrenergic receptor and the PTHR is now well established. How the control of signaling and localization of these GPCRs precisely affects the operation of tissues and organs however remains to be determined. There is now an example where retromer function in regulating a specific GPCR does have profound implications for the operation of a specific tissue - namely the retina of the eye. The rhodopsin1 protein is a GPCR that functions as a light sensor in photoreceptor cells which is endocytosed upon activation by light. Studies in drosophila have shown that, following its endocytosis, the retromer complex is required to mediate the endosome-to-cell surface recycling of rhodopsin1 (Wang et al., 2014). Loss of retromer function results in mistrafficking of activated rhodopsin 1 to a lysosome resulting in its destruction. This, in turn, leads to light-induced degeneration of photoreceptor cells although mechanistically it is not clear why this happens. Additionally, whether the recycling of rhodopsin 1 by retromer also involves a drosophila homologue of SNX27 also remains to be determined but it appears that there is homologue of SNX27 in flies encoded by the CG32758 gene.

In addition to binding to both VPS26 and also the FAM21 subunit of the WASH complex, the SNX27 protein also has the ability to interact with Ras (Ghai et al., 2011). Ras can be considered to be the archetypal small GTPase and is associated with multiple signaling events/pathways that have long been 
implicated in oncogenic signaling mechanisms (for review see Herrero et al., 2016). The SNX27 - Ras interaction occurs through the FERM domain of SNX27 that has also been implicated in binding to cargo proteins containing the NPxY sorting motif (Burden et al., 2004; Ghai et al., 2011). One of the cargo proteins that depends on an intact SNX27 FERM domain for its localization is a class of potassium channels that are important for regulating neuronal excitability - namely the G-protein regulated inward rectifying potassium (GIRK) channels (Balana et al., 2013) although other domains in SNX27 may also be important for potassium channel trafficking (Lunn et al., 2007). In cells expressing a dominant negative Ras protein that cannot bind GTP, the ability of SNX27 to regulate cell surface levels of the GIRK potassium channels was compromised (Balana et al., 2013).

These findings raise some intriguing possibilities and questions: for example, does SNX27 associate with Ras whilst also interacting with retromer (via VPS26) and the WASH complex? Is cargo-binding by SNX27 affected by an interaction between SNX27 and Ras? Does the SNX27 - Ras interaction modulate the role and/or function of Ras in various signaling pathways? Answers to these questions have yet to be reported but there is indeed a rich vein of research to be mined for functional links between the retromercentered endosomal sorting machinery and the Ras protein. Adding to the questions posed by the SNX27 - Ras association is the recent identification of an interaction between the VPS35 component of retromer and N-Ras, a version of the Ras protein that is farnesylated rather than being palmitoylated and is generally found to be cytoplasmic (Zhou et al., 2016). The interaction between retromer and $\mathrm{N}$-Ras appears to be restricted to cytoplasmic retromer which would not be regarded as functional as retromer must be localized to endosomes to operate in endosomal protein sorting. Thus it is possible that the retromer - N-Ras interaction may represent a means of regulating the activity of retromer whilst it is cytoplasmic although this hypothesis has yet to be proven.

\section{Retromer-mediated regulation of neuronal signaling}


SNX27 plays a key role in regulating the trafficking of several membrane proteins than can function as receptors (e.g. rhodopsin, a light receptor) or channels (e.g. the GIRK channels) and does indeed seem to have a preference for multi-membrane spanning proteins as cargo, another notable example being the Glut-1, glucose transporter protein (Steinberg et al., 2014). Indeed it has also been reported that SNX27 mediates the endosome-to-cell surface recycling of the AMPA (a-amino-3-hydroxy-5-methyl-4isoxazolepropionic acid) receptor (Wang et al., 2013; Loo et al., 2014; Hussein et al., 2014) - a receptor for glutamate in neuronal cells that is implicated in long-term potentiation (LTP), a process intricately linked with memory and learning. In a study of AMPA receptor trafficking it was shown that following stimulation of LTP, K-Ras is recruited to endosomes that are enriched in SNX27 and that this is necessary for synaptic delivery of GluA1, a glutamate receptor. Studies in neuronal cells have revealed a role for retromer in trafficking proteins to dendritic spines (Choy et al., 2014) and interestingly, localization and trafficking of GluA1 receptors has been shown to be inhibited in cells expressing a mutant of VPS35 that is responsible for a rare form of inherited Parkinson's disease (PD). The PD-causing allele of VPS35 is a mutation of aspartate at position 620 to glutamate and results in reduced binding of the WASH complex by VPS35 (Zavodszky et al., 2014; McGough et al., 2014) which, in-turn, leads to mistrafficking of many WASH-complex dependent cargo proteins including the Glut-1 glucose transporter and the GluA1 glutamate receptor (Munsie et al., 2015; Tian et al., 2015). It is currently not known how the PD-causing VPS35 D620N mutant might affect the signaling pathways that depend upon the retromer - WASH complex association but it seems likely that several distinct pathways may be affected. Not only does mutation of VPS35 affect transport of AMPA receptors such as GluA1, but loss of SNX27 can also lead to deficient transport of excitatory receptors. In a small number of patients, mutations in SNX27 cause a form of epilepsy associated with additional neurological abnormalities (Damseh et al., 2015). These observations demonstrate the key role that retromer, along with its accessory proteins such as the WASH complex and SNX27, plays in mediating the localization of neuronally important proteins such as the AMPA receptors and thereby illustrates how retromer contributes to signaling events 
that are key to the function of the brain such as the establishment of long-term potentiation.

A role for retromer in regulating bone remodeling

The trafficking of glutamate receptors in neuronal cells is an example of how retromer function can influence relatively rapid signaling events that may, in some circumstances, lead to longer term alterations in cell-cell contacts which can influence processes such as synaptic plasticity. Another example of how retromer can influence signaling that controls large-scale changes is the regulating of the localization and trafficking of the RANK protein by retromer. The RANK (Receptor Activator of NF-кB) protein functions in bone remodeling - osteoclastogenesis - and as such is important in maintaining bone density and structure. Loss of VPS35 function results in mislocalisation of RANK which causes dysregulation of signaling from the RANK ligand and leads to decreased bone formation and a condition similar to osteoporosis (Xia et al., 2013). Precisely how VPS35 (and presumably retromer) contributes to RANK localization has yet to be determined but these data illustrate how important it is for normal signaling to be maintained and the key role that retromer plays in this form of homeostasis.

Retromer regulation of Wnt-mediated morphogenic signaling

Another example of how retromer function can influence signaling events in the longer term or over longer distances is the requirement for retromer to control the localization of the Wntless protein. The secretion of the Wnt morphogen that regulates some key developmental processes requires the Wntless protein - a multi-pass membrane protein that traffics between the cell surface and the TGN. Whilst in the TGN, Wntless can bind to Wnt and then facilitate the secretion of Wnt at the cell surface. Wntless is subsequently endocytosed and then recycled back to the TGN via retromer-mediated endosome-to-Golgi retrieval (Franch-Marro et al., 2008; Port et al., 2008; Belenkaya et al., 2008; Yang et al., 2008; Pan et al., 2008). In this instance, 
retromer operates with another sorting nexin, that like SNX27, is not a SNXBAR protein involved in membrane tubule formation. Here, retromer operates with SNX3, a small sorting nexin that comprises a phosophotidyl inositol 3phosphate binding domain and little more (Harterink et al., 2011; Zhang et al., 2011). SNX3 is conserved and functions with retromer in endosome-to-Golgi retrieval in yeast although it is known as Grd19p in yeast (Strochlic et al., 2007). Failure of recycling of Wntless by retromer leads to reduced Wnt secretion due to a lack of Wntless in the TGN much like loss of retromer leading to reduced TGN localization of the CIMPR which in-turn results in a failure of delivery of lysosomal hydrolases (e.g. Cathepsin D) to the lysosome (see Figure 2). The reduction in Wnt secretion following inhibition of retromer function results in developmental abnormalities.

Retromer-mediated regulation of signaling in the immune system

One of the tissue types often associated with complicated signaling pathways is the immune system - specifically signaling in T-cells in response to the various cytokines. Recently it has been reported that retromer can modulate the signaling response initiated by binding of type-I interferons to their receptor. The type-I family of interferons are typically associated with the response to bacterial or viral infections. Once ligand (i.e. the type-I interferon) has bound to a receptor comprising two subunits (IFNAR1 and IFNAR2), the receptor is internalized through clathrin-mediated endocytosis and delivered to an endosome where signaling via JAK/STAT is initiated. The two receptor subunits then dissociate with the IFNAR2 being recycled to the cell surface whilst the IFNAR1 is directed to the lysosome for degradation. Recycling of IFNAR2 to the cell surface requires retromer (Chmiest et al., 2016). Interestingly, retromer association with IFNAR2 may facilitate the dissociation of the interferon receptor and thereby down regulate the signaling by JAK/STAT as loss of VPS35 leads to increased localization of the IFNAR1 and IFNAR2 proteins at the endosome and prolonged activation of JAK/STAT with increased downstream events as well such as transcription of specific genes associated with the immune response. 
Thus, although at the beginning of this review I suggested that retromer can modulate signaling pathways in very different ways to the mode of action of the ESCRT machinery, there are some similarities depending on the mechanism of action of the proteins that initiate the signaling event. For GPCRs (e.g. the parathyroid hormone receptor), retromer can modulate signaling by displacing the $\beta$-arrestin protein from the activated GPCR thereby silencing the signaling prior to directing the GPCR into an endosome-to-cell surface recycling pathway. But for activated interferon receptors, retromer down regulates the receptor by causing the two subunits to dissociate, one is then degraded, the other recycled.

\section{Concluding remarks}

In conclusion, endosomal protein sorting mediated by the retromer complex along with associated proteins such as the WASH complex and SNX27 has been clearly shown to be critical for trafficking and recycling of membrane proteins from endosomes to the Golgi and also from endosomes to the cell surface. There are now numerous examples of how retromer contributes to regulating signaling events by controlling the trafficking and localization of membrane proteins that initiate signaling events. In some instances, retromer can directly participate in the signaling activity of a pathway, for example when the VPS26 subunit of retromer displaces the $\beta$-arrestin from an activated GPCR thereby bringing to a halt the signaling pathway initiated when the GPCR was activated. In other reports, retromer has been shown to control the cell surface localization of important neuronal receptors, (e.g. GluA1) and hence retromer function contributes to signaling events that are instrumental in neuronal processes such as long-term potentiation. Retromer may also indirectly affect signaling pathways by regulating the localization of a protein that is involved in secretion of a morphogen that operates over relatively long distances and time scale - as is the case for the Wnt morphogen and the influence that retromer plays in its secretion via the Wntless protein. 
It seems likely that further examples of how retromer function contributes to the regulation of signaling pathways and events that may be initiated at the cell surface but are modulated at endosomal membrane will be reported in the near future. For cell biologists interested in understanding how protein localization contributes to signaling, these are interesting and exciting times.

\section{Acknowledgements}

I would like to thank Aamir Mukadam for critical reading of the manuscript. MNJS is funded by the Medical Research Council.

\section{References}

Arighi CN, Hartnell LM, Aguilar RC, Haft CR, Bonifacino JS. (2004). Role of the mammalian retromer in sorting of the cation-independent mannose 6phosphate receptor. J Cell Biol. 165(1):123-33.

Aubry L, Klein G. (2013). True arrestins and arrestin-fold proteins: a structurebased appraisal. Prog Mol Biol Transl Sci.118:21-56.

Balana B, Bahima L, Bodhinathan K, Taura JJ, Taylor NM, Nettleton MY, Ciruela F, Slesinger PA. (2013). Ras-association domain of sorting Nexin 27 is critical for regulating expression of GIRK potassium channels. PLoS One. 8(3):e59800.

Belenkaya TY, Wu Y, Tang X, Zhou B, Cheng L, Sharma YV, Yan D, Selva EM, Lin X. (2008). The retromer complex influences Wnt secretion by recycling wntless from endosomes to the trans-Golgi network. Dev Cell. 14(1):120-31.

Burden JJ, Sun XM, García AB, Soutar AK. (2004). Sorting motifs in the intracellular domain of the low density lipoprotein receptor interact with a novel domain of sorting nexin-17. J Biol Chem. 279(16):16237-45.

Carlton J, Bujny M, Peter BJ, Oorschot VM, Rutherford A, Mellor H, 
Klumperman J, McMahon HT, Cullen PJ. (2004). Sorting nexin-1 mediates tubular endosome-to-TGN transport through coincidence sensing of highcurvature membranes and 3-phosphoinositides.

Curr Biol. 14(20):1791-800.

Chan AS, Clairfeuille T, Landao-Bassonga E, Kinna G, Ng PY, Loo LS, Cheng TS, Zheng M, Hong W, Teasdale RD, Collins BM, Pavlos NJ. (2016).

Sorting nexin 27 couples PTHR trafficking to retromer for signal regulation in osteoblasts during bone growth. Mol Biol Cell. 27(8):1367-82.

Chmiest D, Sharma N, Zanin N, Viaris de Lesegno C, Shafaq-Zadah M, Sibut V, Dingli F, Hupé P, Wilmes S, Piehler J, Loew D, Johannes L, Schreiber G, Lamaze C. (2016). Spatiotemporal control of interferon-induced JAK/STAT signaling and gene transcription by the retromer complex. Nat Commun. 7:13476.

Choy RW, Park M, Temkin P, Herring BE, Marley A, Nicoll RA, von Zastrow M. (2014). Retromer mediates a discrete route of local membrane delivery to dendrites. Neuron. 82(1):55-62.

Collins BM, Norwood SJ, Kerr MC, Mahony D, Seaman MN, Teasdale RD, Owen DJ. (2008). Structure of Vps26B and mapping of its interaction with the retromer protein complex. Traffic. 9(3):366-79.

Damseh N, Danson CM, Al-Ashhab M, Abu-Libdeh B, Gallon M, Sharma K, Yaacov B, Coulthard E, Caldwell MA, Edvardson S, Cullen PJ, Elpeleg O. (2015). A defect in the retromer accessory protein, SNX27, manifests by infantile myoclonic epilepsy and neurodegeneration. Neurogenetics. 16(3):215-21.

Derivery E, Sousa C, Gautier JJ, Lombard B, Loew D, Gautreau A. (2009). The Arp2/3 activator WASH controls the fission of endosomes through a large multiprotein complex. Dev Cell. 17(5):712-23. 
Feinstein TN, Wehbi VL, Ardura JA, Wheeler DS, Ferrandon S, Gardella TJ, Vilardaga JP. (2011). Retromer terminates the generation of cAMP by internalized PTH receptors. Nat Chem Biol. 7(5):278-84.

Franch-Marro X, Wendler F, Guidato S, Griffith J, Baena-Lopez A, Itasaki N, Maurice MM, Vincent JP. (2008). Wingless secretion requires endosome-toGolgi retrieval of Wntless/Evi/Sprinter by the retromer complex. Nat Cell Biol. 10(2):170-7

Freeman CL, Hesketh G, Seaman MN. (2014). RME-8 coordinates the activity of the WASH complex with the function of the retromer SNX dimer to control endosomal tubulation. J Cell Sci. 127(Pt 9):2053-70.

Gallon M, Clairfeuille T, Steinberg F, Mas C, Ghai R, Sessions RB, Teasdale RD, Collins BM, Cullen PJ. (2014). A unique PDZ domain and arrestin-like fold interaction reveals mechanistic details of endocytic recycling by SNX27retromer. Proc Natl Acad Sci U S A. 111(35):E3604-13.

Ghai R, Mobli M, Norwood SJ, Bugarcic A, Teasdale RD, King GF, Collins BM. (2011). Phox homology band 4.1/ezrin/radixin/moesin-like proteins function as molecular scaffolds that interact with cargo receptors and Ras GTPases. Proc Natl Acad Sci U S A. 108(19):7763-8.

Gurevich VV, Gurevich EV. (2014). Overview of different mechanisms of arrestin-mediated signaling. Curr Protoc Pharmacol. 1;67.

Haft CR, de la Luz Sierra M, Bafford R, Lesniak MA, Barr VA, Taylor SI. (2000). Human orthologs of yeast vacuolar protein sorting proteins Vps26, 29, and 35: assembly into multimeric complexes. Mol Biol Cell. 11(12):4105-16.

Harbour ME, Breusegem SY, Seaman MN. (2012). Recruitment of the endosomal WASH complex is mediated by the extended 'tail' of Fam21 binding to the retromer protein Vps35. Biochem J. 442(1):209-20. 
Harbour ME, Seaman MN. (2011) Evolutionary variations of VPS29, and their implications for the heteropentameric model of retromer. Commun Integr Biol. 2011 Sep;4(5):619-22.

Harterink M, Port F, Lorenowicz MJ, McGough IJ, Silhankova M, Betist MC, van Weering JR, van Heesbeen RG, Middelkoop TC, Basler K, Cullen PJ, Korswagen HC. (2011). A SNX3-dependent retromer pathway mediates retrograde transport of the Wnt sorting receptor Wntless and is required for Wnt secretion. Nat Cell Biol. 13(8):914-23.

Helfer E, Harbour ME, Henriot V, Lakisic G, Sousa-Blin C, Volceanov L, Seaman MN, Gautreau A. (2013). Endosomal recruitment of the WASH complex: active sequences and mutations impairing interaction with the retromer. Biol Cell. 105(5):191-207.

Herrero A, Matallanas D, Kolch W. (2016). The spatiotemporal regulation of RAS signaling. Biochem Soc Trans. 44(5):1517-1522.

Hussain NK, Diering GH, Sole J, Anggono V, Huganir RL. (2014). Sorting Nexin 27 regulates basal and activity-dependent trafficking of AMPARs. Proc Natl Acad Sci U S A. 111(32):11840-5.

Jia D, Gomez TS, Billadeau DD, Rosen MK. (2012). Multiple repeat elements within the FAM21 tail link the WASH actin regulatory complex to the retromer. Mol Biol Cell. 23(12):2352-61.

Lee S, Chang J, Blackstone C. (2016). FAM21 directs SNX27-retromer cargoes to the plasma membrane by preventing transport to the Golgi apparatus. Nat Commun. 9;7:10939.

Loo LS, Tang N, Al-Haddawi M, Dawe GS, Hong W. (2014). A role for sorting nexin 27 in AMPA receptor trafficking. Nat Commun. 5:3176.

Lunn ML, Nassirpour R, Arrabit C, Tan J, McLeod I, Arias CM, Sawchenko 
PE, Yates JR 3rd, Slesinger PA. (2007). A unique sorting nexin regulates trafficking of potassium channels via a PDZ domain interaction. Nat Neurosci. 10(10):1249-59.

McGarvey JC, Xiao K, Bowman SL, Mamonova T, Zhang Q, Bisello A, Sneddon WB, Ardura JA, Jean-Alphonse F, Vilardaga JP, Puthenveedu MA, Friedman PA. (2016). Actin-Sorting Nexin 27 (SNX27)-Retromer Complex Mediates Rapid Parathyroid Hormone Receptor Recycling. J Biol Chem. 291(21):10986-1002.

McGough IJ, Steinberg F, Jia D, Barbuti PA, McMillan KJ, Heesom KJ, Whone AL, Caldwell MA, Billadeau DD, Rosen MK, Cullen PJ. (2014). Retromer binding to FAM21 and the WASH complex is perturbed by the Parkinson disease-linked VPS35(D620N) mutation. Curr Biol. 24(14):1670-6.

Munsie LN, Milnerwood AJ, Seibler P, Beccano-Kelly DA, Tatarnikov I, Khinda J, Volta M, Kadgien C, Cao LP, Tapia L, Klein C, Farrer MJ (2015). Retromerdependent neurotransmitter receptor trafficking to synapses is altered by the Parkinson's disease VPS35 mutation p.D620N. Hum Mol Genet. 24(6):1691703.

Pan CL, Baum PD, Gu M, Jorgensen EM, Clark SG, Garriga G. (2008). C. elegans AP-2 and retromer control Wnt signaling by regulating mig14/Wntless. Dev Cell. 14(1):132-9.

Port F, Kuster M, Herr P, Furger E, Bänziger C, Hausmann G, Basler K. (2008). Wingless secretion promotes and requires retromer-dependent cycling of Wntless. Nat Cell Biol. 10(2):178-85.

Schuh AL, Audhya A. The ESCRT machinery: from the plasma membrane to endosomes and back again. (2014). Crit Rev Biochem Mol Biol. 49(3):242-61.

Seaman MN. (2004). Cargo-selective endosomal sorting for retrieval to the Golgi requires retromer. J Cell Biol. 165(1):111-22. 
Seaman MN. (2012). The retromer complex - endosomal protein recycling and beyond. J Cell Sci. 125(Pt 20):4693-702.

Seaman MN, Gautreau A, Billadeau DD. (2013). Retromer-mediated endosomal protein sorting: all WASHed up! Trends Cell Biol. 23(11):522-8.

Seaman MN, Marcusson EG, Cereghino JL, Emr SD. (1997) Endosome to Golgi retrieval of the vacuolar protein sorting receptor, Vps10p, requires the function of the VPS29, VPS30, and VPS35 gene products. J Cell Biol. 137(1):79-92.

Seaman MN, McCaffery JM, Emr SD. (1998) A membrane coat complex essential for endosome-to-Golgi retrograde transport in yeast. J Cell Biol. 142(3):665-81.

Shi H, Rojas R, Bonifacino JS, Hurley JH. (2006). The retromer subunit Vps26 has an arrestin fold and binds Vps35 through its C-terminal domain. Nat Struct Mol Biol. 13(6):540-8.

Steinberg F, Gallon M, Winfield M, Thomas EC, Bell AJ, Heesom KJ, Tavaré JM, Cullen PJ. (2014). A global analysis of SNX27-retromer assembly and cargo specificity reveals a function in glucose and metal ion transport. Nat Cell Biol. 15(5):461-71.

Strochlic TI, Setty TG, Sitaram A, Burd CG. (2007). Grd19/Snx3p functions as a cargo-specific adapter for retromer-dependent endocytic recycling. J Cell Biol. 177(1):115-25.

Swarbrick JD, Shaw DJ, Chhabra S, Ghai R, Valkov E, Norwood SJ, Seaman MN, Collins BM. (2011). VPS29 is not an active metallo-phosphatase but is a rigid scaffold required for retromer interaction with accessory proteins. PLoS One. 6(5):e20420. 
Temkin P, Lauffer B, Jäger S, Cimermancic P, Krogan NJ, von Zastrow M. (2011). SNX27 mediates retromer tubule entry and endosome-to-plasma membrane trafficking of signaling receptors. Nat Cell Biol. 13(6):715-21.

Tian Y, Tang FL, Sun X, Wen L, Mei L, Tang BS, Xiong WC. (2015). VPS35deficiency results in an impaired AMPA receptor trafficking and decreased dendritic spine maturation. Mol Brain. 8(1):70.

van Weering JR, Cullen PJ. (2014). Membrane-associated cargo recycling by tubule-based endosomal sorting. Semin Cell Dev Biol. 31:40-7.

Wang S, Tan KL, Agosto MA, Xiong B, Yamamoto S, Sandoval H, Jaiswal M, Bayat V, Zhang K, Charng WL, David G, Duraine L, Venkatachalam K, Wensel TG, Bellen HJ. (2014). The retromer complex is required for rhodopsin recycling and its loss leads to photoreceptor degeneration. PLoS Biol. 12(4):e1001847.

Wang X, Zhao Y, Zhang X, Badie H, Zhou Y, Mu Y, Loo LS, Cai L, Thompson RC, Yang B, Chen Y, Johnson PF, Wu C, Bu G, Mobley WC, Zhang D, Gage FH, Ranscht B, Zhang YW, Lipton SA, Hong W, Xu H. (2013). Loss of sorting nexin 27 contributes to excitatory synaptic dysfunction by modulating glutamate receptor recycling in Down's syndrome. Nat Med. 19(4):473-80.

Xia WF, Tang FL, Xiong L, Xiong S, Jung JU, Lee DH, Li XS, Feng X, Mei L, Xiong WC. (2013). Vps35 loss promotes hyperresorptive osteoclastogenesis and osteoporosis via sustained RANKL signaling. J Cell Biol. 200(6):821-37.

Yang PT, Lorenowicz MJ, Silhankova M, Coudreuse DY, Betist MC, Korswagen HC. (2008) Wnt signaling requires retromer-dependent recycling of MIG-14/Wntless in Wnt-producing cells. Dev Cell. 14(1):140-7.

Zavodszky E, Seaman MN, Moreau K, Jimenez-Sanchez M, Breusegem SY, Harbour ME, Rubinsztein DC. (2014). Mutation in VPS35 associated with Parkinson's disease impairs WASH complex association and inhibits 
autophagy. Nat Commun. 5:3828.

Zhang P, Wu Y, Belenkaya TY, Lin X. (2011). SNX3 controls Wingless/Wnt secretion through regulating retromer-dependent recycling of Wntless. Cell Res. 21(12):1677-90.

Zhou M, Wiener H, Su W, Zhou Y, Liot C, Ahearn I, Hancock JF, Philips MR. (2016). VPS35 binds farnesylated N-Ras in the cytosol to regulate N-Ras trafficking. J Cell Biol. 214(4):445-58.

\section{Figure legends}

Figure 1.

Schematic of the retromer complex and key accessory proteins. The retromer complex comprises two functional units; a cargo-selective trimer of VPS35, VPS29 and VPS26 (often referred to as the cargo-selective complex - CSC) and a membrane bending/tubulating dimer of SNX1 or SNX2 with either SNX5 or SNX6. The VPS26 protein is structurally related to $\beta$-arrestin and can modulate GPCR signaling at endosomes. SNX27 interacts with both VPS26 and the FAM21 subunit of the WASH complex that promotes F-actin formation on endosomes. The PDZ and FERM domains of SNX27 both play important roles in trafficking membrane proteins (including those involved in signaling) from endosomes to the cell surface. The SNX27 PX domain binds to phosphotidyl inositol 3-phosphate (Ptdlns-3P) in endosomal membranes.

Figure 2.

Involvement of retromer and accessory proteins in trafficking pathways from endosomes. The retromer cargo-selective complex (CSC) along with SNX27 functions to sort proteins into tubular carriers for the endosome-to-cell surface recycling pathway. Cargo proteins sorted by retromer with SNX27 include GPCRs where the VPS26 subunit of retromer can displace $\beta$-arrestin from the cytoplasmic tail of the GPCR. Other membrane proteins involved in signaling that rely on retromer include the interferon receptor subunit, IFNAR2. At the endsosome, retromer causes the IFNAR1 and IFNAR2 proteins to dissociate 
and directs the IFNAR2 protein back to the cell surface whilst the IFNAR1 will be degraded in the lysosome. Retromer also functions in endosome-to-Golgi retrieval and maintains a pool of receptors in the TGN including Wntless, the morphogen receptor required for Wnt secretion. 


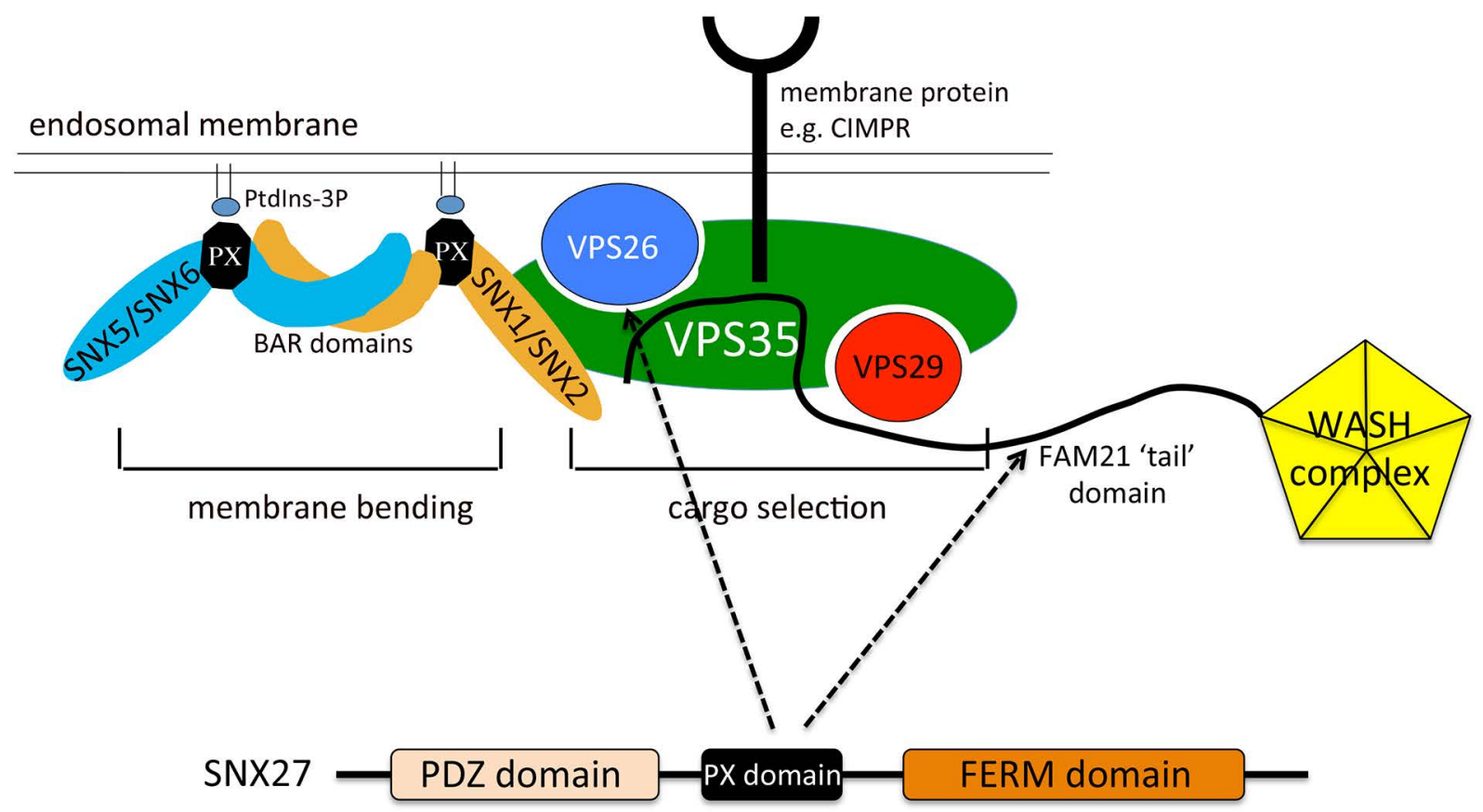

Figure 1 


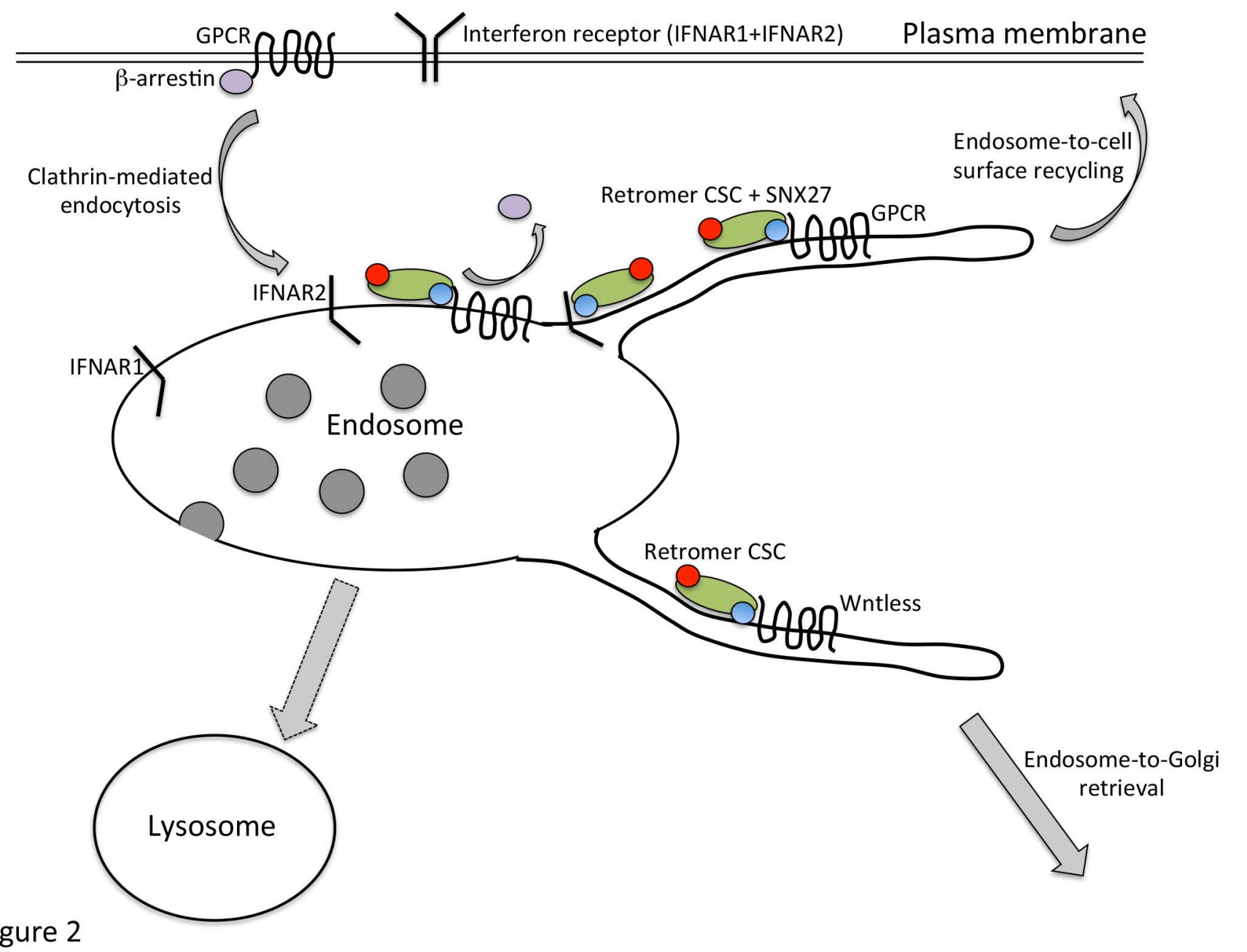

